\title{
Erratum: Towards storage rings as quantum computers [Phys. Rev. Accel. Beams 23, 054701 (2020)]
}

\author{
K. A. Brown $\odot$ and T. Roser
}

(Received 21 March 2021; published 16 April 2021)

DOI: 10.1103/PhysRevAccelBeams.24.049901

In this paper, Eq. (3) is incorrect. A correct expression is [1],

$$
T_{\|}[K]=\left(\frac{2}{k_{B}}\right)\left(\frac{\delta p}{p_{0}}\right)^{2} E_{0}[\mathrm{eV}] .
$$

This correction does not require any changes in the rest of the paper except for quantities given in the text and Fig. 3 of the paper that are based on Eq. (3). These changes are listed below: (1) Section VI, paragraph 5: sentence 5 should read "... the product $\left(\frac{\delta p}{p_{0}}\right)^{2} E_{0}=2.3 \times 10^{-5} / 2.32 \times 10^{4} \approx 1 \times 10^{-9}$." (2) Section VI, paragraph 5: sentence 6 should read "A beam with $E_{0}=1[\mathrm{eV}]$, corresponding to a velocity of $2818 \mathrm{~m} / \mathrm{s}$, would have to be cooled to have a $\delta p / p_{0}=3.16 \times 10^{-5} \ldots$ " (3) Section VI, paragraph 5: sentence 7 should read “... a beam with $E_{0}=10^{-4}[\mathrm{eV}]$, with a velocity near $28 \mathrm{~m} / \mathrm{s}$, would

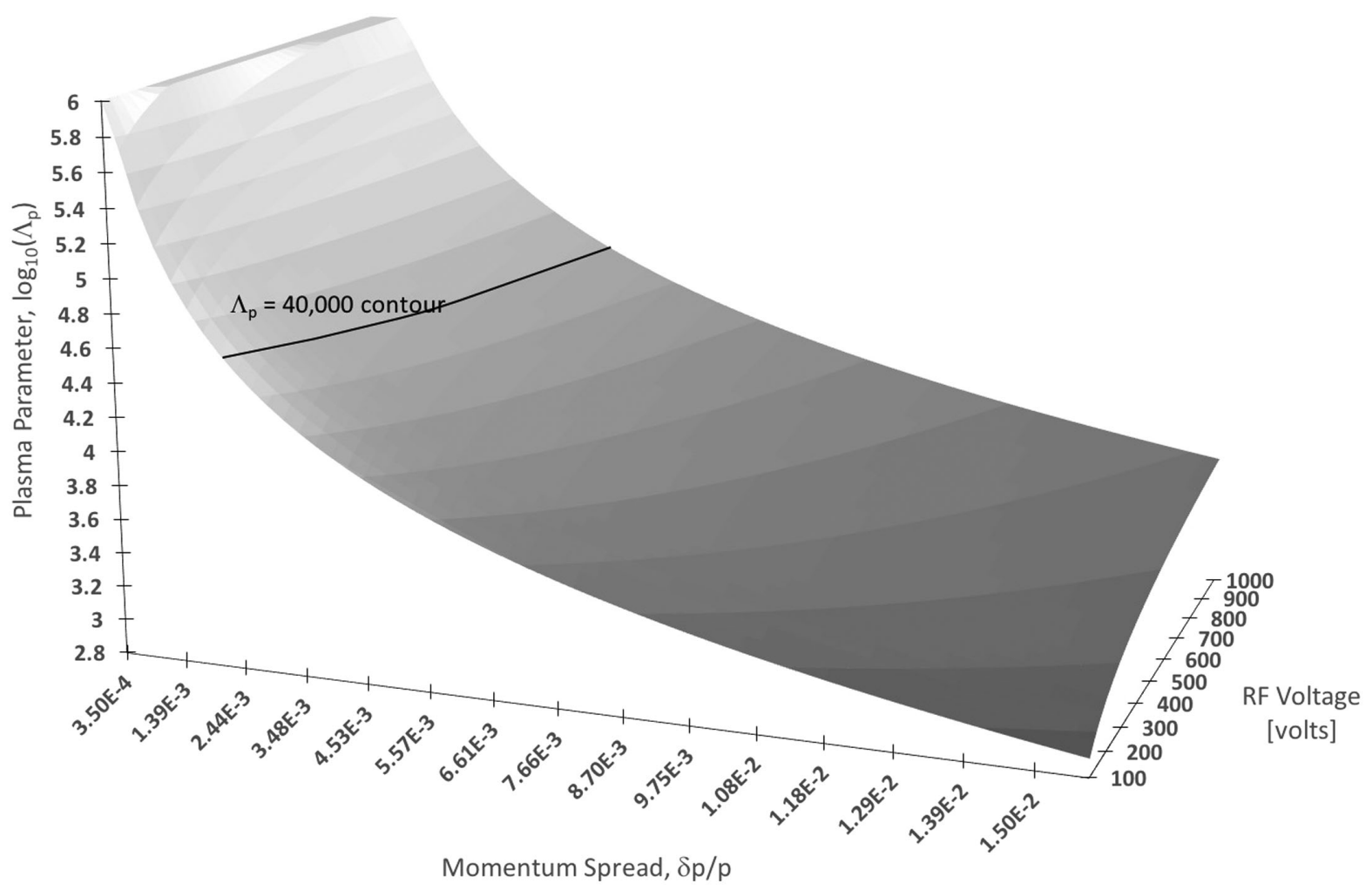

FIG. 3. The surface of values of the $\log _{10} \Lambda_{p}$ versus momentum spread and voltage for ${ }^{7} \mathrm{Li}^{+}$ions with $v_{0}=100 \mathrm{~m} / \mathrm{s}$, at $f_{\text {rf }}=12.6 \mathrm{Mhz}$.

Published by the American Physical Society under the terms of the Creative Commons Attribution 4.0 International license. Further distribution of this work must maintain attribution to the author(s) and the published articles title, journal citation, and DOI. 
have to be cooled to have a $\delta p / p_{0}=3.16 \times 10^{-3}$." (4) Section VI, paragraph 6: With respect to Table 1, sentence 2 should read "The assumption is we can cool the beams to a level where the $\delta p / p_{0}$ is $3.16 \times 10^{-4}$." (5) Table 1 caption: "... assume cooling has achieved $\delta p / p_{0}$ of $3.16 \times 10^{-4}$." (6) Section VI, paragraph 11: sentence 3 should read "For the $v=100 \mathrm{~m} / \mathrm{s}$ ions, the range of $\delta p / p_{0}$ must be less than $3.16 \times 10^{-3}$." (7) Section VI, paragraph 14: sentence 2 should read "The laser cooling will need to reduce the $\delta p / p_{0}$ to less than $3.16 \times 10^{-3}$."

Included is an updated version of Fig. 3, with correct parameter ranges.

With this change in the fundamental relation between longitudinal temperature and $\delta p / p_{0}$, numerical values of quantities change in directions that make it easier to reach the conditions for quantum entanglement.

This correction does not change the thesis or conclusions of the paper.

\section{ACKNOWLEDGMENTS}

We wish to thank our colleague who identified this error. This work was performed under Contract No. DE-SC0012704 with the auspices of the U.S. Department of Energy.

[1] M. Steck, K. Beckert, H. Eickhoff, B. Franzke, F. Nolden, H. Reich, B. Schlitt, and T. Winkler, Anomalous Temperature Reduction of Electron-Cooled Heavy Ion Beams in the Storage Ring ESR, Phys. Rev. Lett. 77, 3803 (1996). 